\title{
Analytical study of on-chip generations of analog sine-wave based on combined digital signals
}

\author{
David-Grignot Stephane ${ }^{1}$, Lamlih Achraf ${ }^{1}$, Kerzerho Vincent ${ }^{1}$, Azaıs Florence ${ }^{1}$, Soulier Fabien ${ }^{1}$, \\ Bernard Serge ${ }^{1}$, Rouyer Tristan ${ }^{2}$, Bonhommeau Sylvain ${ }^{2}$
}

${ }_{2}^{1}$ University of Montpellier 161 rue Ada 34095 Montpellier Cedex 5, France

2 Ifremer/MARBEC, Av. J. Monnet, 34203 Sete, France

Stephane.David-grignot@lirmm.fr ; Achraf.Lamlih@lirmm.fr ; Vincent.Kerzerho@lirmm.fr ; Florence.Azais@lirmm.fr ; Fabien.Soulier@lirmm.fr ; Serge.Bernard@lirmm.fr ; Tristan.Rouyer@ifremer.fr ; Sylvain.Bonhommeau@ifremer.fr

\begin{abstract}
:
On-chip sine-wave signal generation is widely covered by literature for Built-In-Self Test (BIST) or biosensor applications. The objective is to generate pure and robust sinewave signal with minimal hardware resources. An attractive solution consists in combining several digital signals to built this analog sine-wave. The objective of this paper is to give an analytical study of various potential solutions based on digitalbased approaches. Thanks to this study, we prove that technique consisting in setting the phase shifts and various amplitude values of the square-wave signals is the most efficient approach. Moreover, this study allows the selection of the best solution in terms of parameters of the square-wave signals to cancel loworder harmonics of the generated signal.
\end{abstract}

Keywords : Harmonic cancellation, sine-wave generation, on-chip signal generation 


\section{INTRODUCTION}

The design of integrated sine-wave signal generator is a long-term research topic. Such designs have several applications from the Built-In-Self Test (BIST) [1] to impedance spectroscopy for biosensor application [2].

The main objective of designing an integrated sine-wave signal generator is to overcome the need for off-chip generation and driving the signal to the internal node. The constraints for designing such integrated generator are the size and the accuracy of the generated sine-wave signal. In order to minimize the size of the design and its sensitivity to manufacturing process variation, it is relevant to use digital resources to generate the sine-wave signal.

An initial solution, presented by figure 1 consists in generating the targeted sine-wave signal by summing square-wave signals. Unfortunately this solution can induce some glitches at squarewave signal transitions.

A more-advanced solution is presented by figure 2 [1] [2] [3] [4] [5] [6] [7] . It consists in a digital block that generates some square-wave signals with different characteristics (amplitude and/or relative phase). Then, these signals are provided to another block that operates a clever summing, aiming at cancelling some harmonics of the output signal. As squarewave signals have harmonics which amplitudes decrease by $1 / k$ ( $k$ is the order of the harmonic). The basic principle of harmonic cancelling is to sum some square-wave signals with different characteristics in order to sum the fundamental frequency bins and to cancel the harmonics. This process

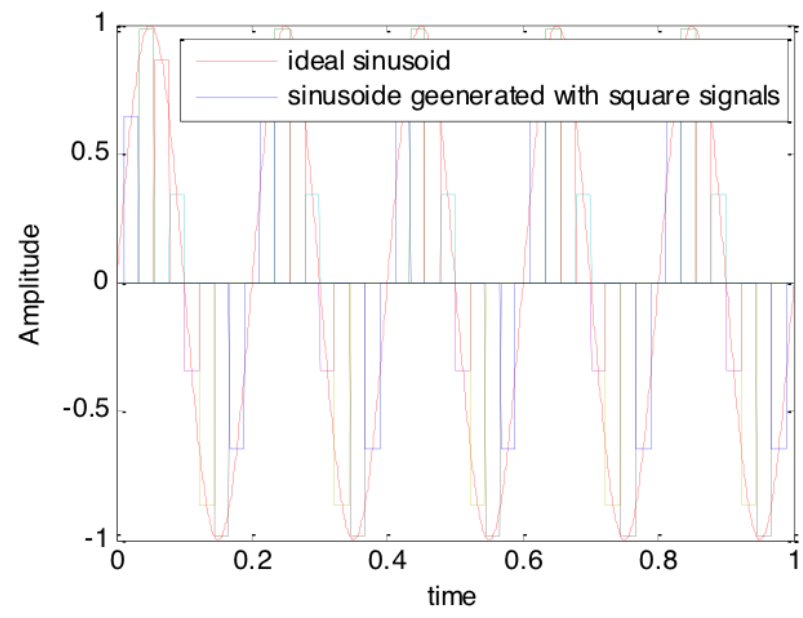

Fig. 1. Quantified sine-wave signal for generation using square-wave signals

can be operated for low-order harmonic cancellation. Then, a generic low-pass filter is used to remove residual highfrequency harmonics.

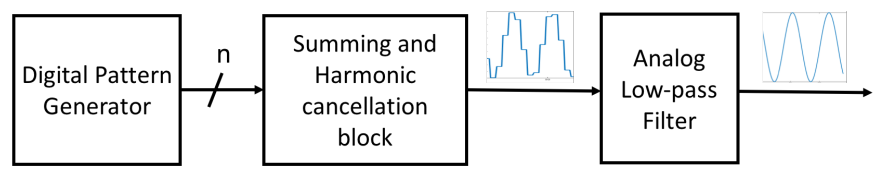

Fig. 2. Basic block diagram of low-distortion sine-wave generation using harmonic cancellation

In order to implement such solution, there are several parameters to set for the generation of the square-wave signals such as their amplitudes, their frequencies, their number and their relative phase-shifts. The architectures described in the literature are strongly application-driven solutions. As a consequence, there is no theoretical analysis of the optimized values of the square-wave signal parameters.

In this paper we propose an analytical study of squarewave parameters for efficient sine-wave generation. This study focuses on performances in terms of linearity of the generated sine-wave, while keeping in mind the consequences on the implementation.

In the second section, we define the parameters of the square- 
wave signals that can be tuned in order to operate the harmonic cancellation. In addition the two main solutions are given. The third section provides the analytical study of harmonic cancellation for the different solutions. A study of robustness of the better solution is provided in the fourth section.

\section{BASIC PRINCIPLE AND CONSTRAINTS}

\section{A. Square-wave signal adjustable parameters}

In order to play with the distortion of the generated sinewave signal, there are several square-wave signal parameters that can be tuned. These parameters are the amplitude, the phase, the duty cycle and the number of square-wave signal $M$. For each square-wave signal $n$, we define its parameters by $A_{n}$ its amplitude, $\varphi_{n}$ its phase and $r_{n}$ its duty cycle. In order to optimize the harmonics cancellation, for $M$ squarewave signals, it makes $3 M$ parameters to study. Then we will present some simplifications.

\section{B. Symmetry of generated square-wave signals}

As presented by figure3, using $M$ square-wave signals to generate the sine-wave signal,if $M$ is an even number, $M / 2$ signals have symmetrical phase shift compare to the other $M / 2$ signals. In case of odd number of square-wave signals, $m=0$ signal is in phase with the targeted sine-wave signal. Then $p=(M-1) / 2$ signals have a symmetrical phase shift compared with the other $p$ signals. Then, we define the squarewave signal names $m$ going from $-p$ to $p$ with $A_{m}=A_{-m}$ and $\varphi_{m}=-\varphi_{-m}$.

The duty cycle can be set aside. Indeed two square-wave signals with a $\varphi_{m}$ symmetrical phase shift are two signals with a duty cycle of $r_{m}=\frac{\pi-2 \varphi_{m}}{2}$. As a consequence any duty cycle can be generated with symmetrical signals.

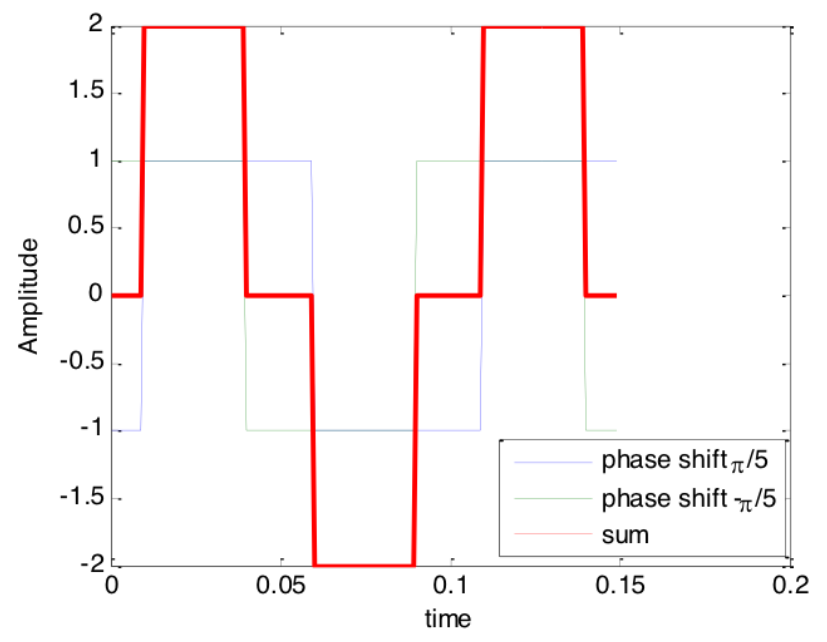

Fig. 3. Symmetry of sine-wave signal generated by summing square-wave signals

\section{Constraints resulting from implementation and open ques- tions}

As previously mentioned, implementation has an impact on the accuracy and robustness of an architecture. Then, from an integration point of view, it is easier to generate a phase shift as a fraction of the sine-wave period rather an arbitrary phaseshift. In addition it is easier to generate signals with identical amplitudes rather than with arbitrary amplitudes.

Considering implementation impacts on accuracy and robustness of an architecture is relevant, but the linearity of the generated signal is also strongly related to the chosen steps for the generated signal. Then some questions remain:

- Is it efficient to strongly constrain the phase-shift of the square-wave signals and precisely adjust their amplitude?

- Is it more efficient to keep one amplitude for any squarewave signal and adjust precisely their relative phase-shift?

- Is adjusting precisely their phase-shift and amplitude the ultimate solution?

These different case studies will be discussed in the following sections

\section{HARMONIC CANCELLATION}

\section{A. Varying amplitude of square-waves}

As described in [7], a possible solution for harmonic cancellation is to set the $\varphi_{m}$ phase-shifts and adjust $A_{m}$ amplitudes.

$$
\left\{\begin{array}{l}
\varphi_{m}=2 \pi \frac{m}{N} \\
A_{m}=\cos \left(2 \pi \frac{m}{N}\right)
\end{array}\right.
$$

with $N=4(p+1)$ and $m=\llbracket-p, p \rrbracket$

The equation 1 presents the phase shifts $\varphi_{m}$ that are a fraction of the sine-wave period and the amplitudes $A_{m}$ that are different for each square-wave signal $m$.

The equation 2 provides the Fourier series description of the sum of the $M$ square-wave signals.

$$
y(t)=\sum_{m=-p}^{p} \sum_{k=-\infty}^{\infty} C_{k, m} e^{i k \omega t}
$$

The Fourier coefficients given by the equation 3 are the sum of the Fourier coefficients of the square-wave signals.

$$
C_{k}(y)=\sum_{m=-p}^{p} \frac{\cos \left(2 \pi \frac{m}{N}\right)}{k \pi}\left(1-e^{i k \pi}\right) e^{-i k m 2 \frac{2 \pi}{N}}
$$

The equation 4 is the development of equation 3.

$$
\begin{gathered}
C_{k}(y)=\frac{2}{k \pi} \sum_{m=-p}^{p} \frac{e^{2 \pi \frac{m}{N}}+e^{-2 \pi \frac{m}{N}}}{2} e^{-i k m 2 \frac{2 \pi}{N}} \\
=\frac{2}{k \pi} \sum_{m=-p}^{p} \frac{e^{2 \pi \frac{m}{N}(k-1)}+e^{-2 \pi \frac{m}{N}(k+1)}}{2}, \text { for odd } \mathrm{k}
\end{gathered}
$$

Considering that the sum of the roots of unity is zero. We can observe that $\sum_{m=-p}^{p} e^{-2 \pi \frac{m}{N}(k+1)}$ cancels when $k$ is not equal to $N-1$. This implies that $C_{k}(y)$ is equal to zero except for $k=1,4(p+1)-1,4(p+1)+1,8(p+1)-1,8(p+1)+1$ ...etc 
Then we demonstrate that thanks to this approach harmonics below $4(p+1)-1$ are cancelled.

Figures 4 and 5 respectively provide the temporal and spectral representation of a sine-wave signal for $p=2$.

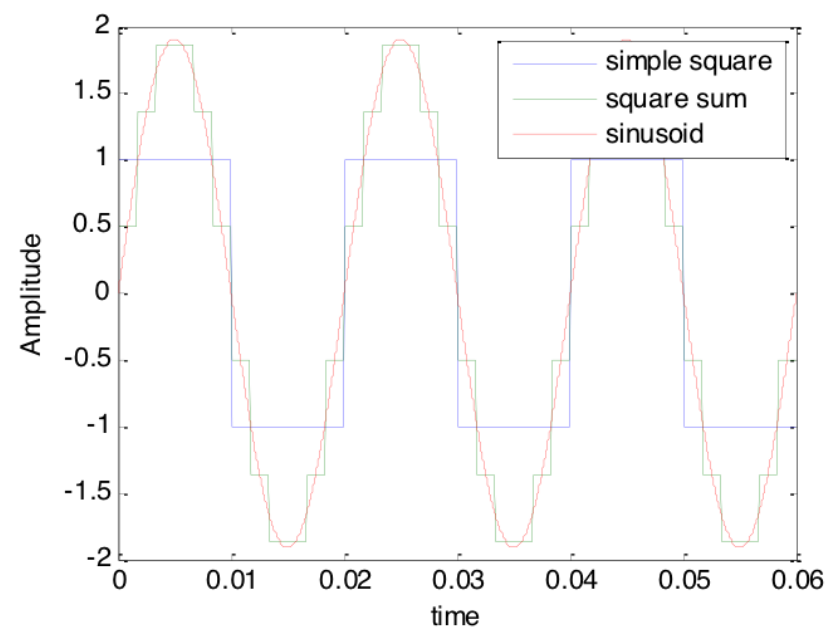

Fig. 4. Sine-wave signal generated using square-wave signals with various amplitudes for $\mathrm{p}=2$

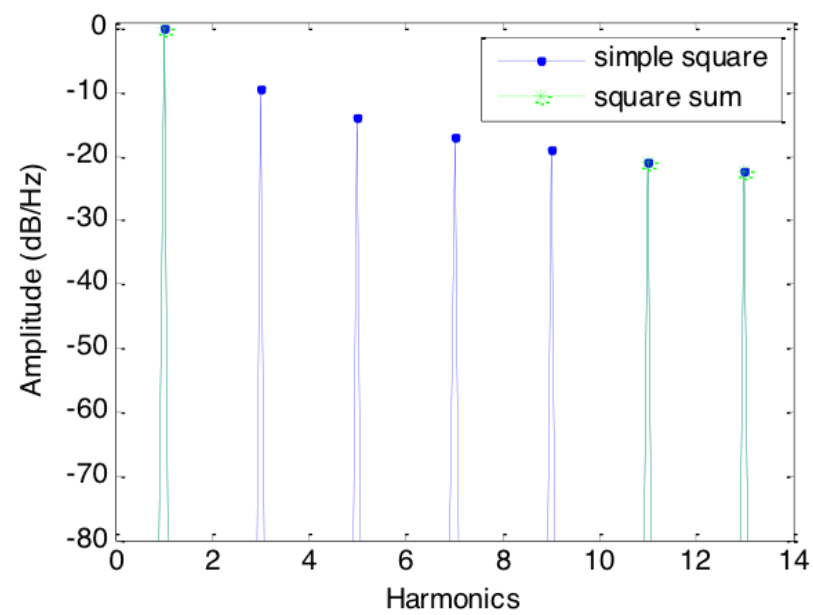

Fig. 5. Spectrum of sine-wave signal generated using square-wave signals with various amplitudes for $\mathrm{p}=2$

\section{B. Varying phase of square-waves}

The opposite solution to the one described in the previous section consists in setting the amplitudes to the same value and adjusting the phase-shifts to cancel the harmonics.

$$
\left\{\begin{array}{l}
\varphi_{m} \text { adjustable } \\
A_{m}=1
\end{array}\right.
$$

Let us consider the Fourier coefficients of the generated signal:

$$
C_{k}(y)=\sum_{m=-p}^{p} \frac{-i}{k \pi} e^{-i k \varphi_{m}}, \text { for odd } \mathrm{k}
$$

To define the values of the $\varphi_{m}$ phase-shifts, we propose to minimize the harmonic distortion ratio provided by equation 7

$$
H D_{N_{h a r}}=\frac{\sqrt{\sum_{m=2}^{N_{h a r}}\left|C_{k}\right|^{2}}}{\left|C_{1}\right|}
$$

For the first integer values of $N_{\text {har }}$ we run a search for a minimum on $H D_{N_{h a r}}$ with equation7. We observe that it is possible to cancel $H D_{N_{h a r}}$ when $N_{h a r}$ is below $2 p+3$.

Figures 6 and 7 respectively provide the temporal and spectral representation of a sine-wave signal generated for $\mathrm{p}=3$

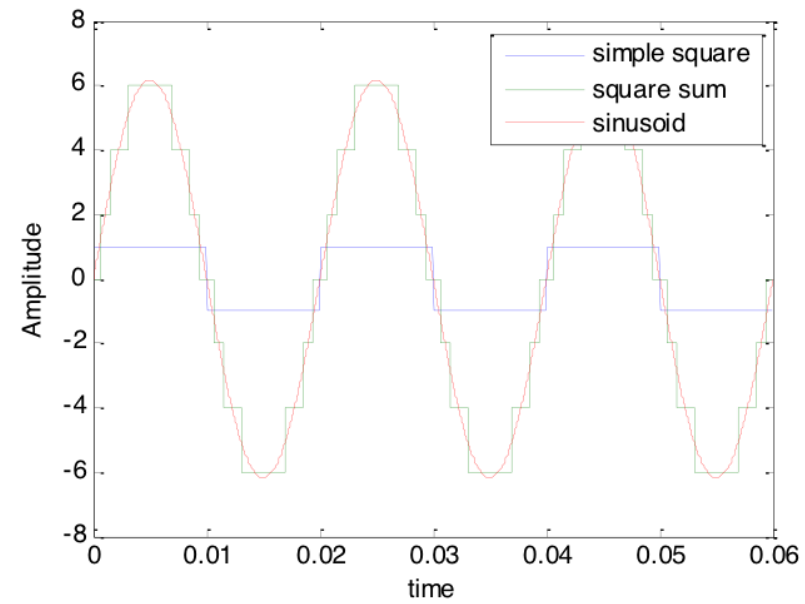

Fig. 6. Sine-wave signal generated using square-wave signals with various phase shifts for $\mathrm{p}=2$

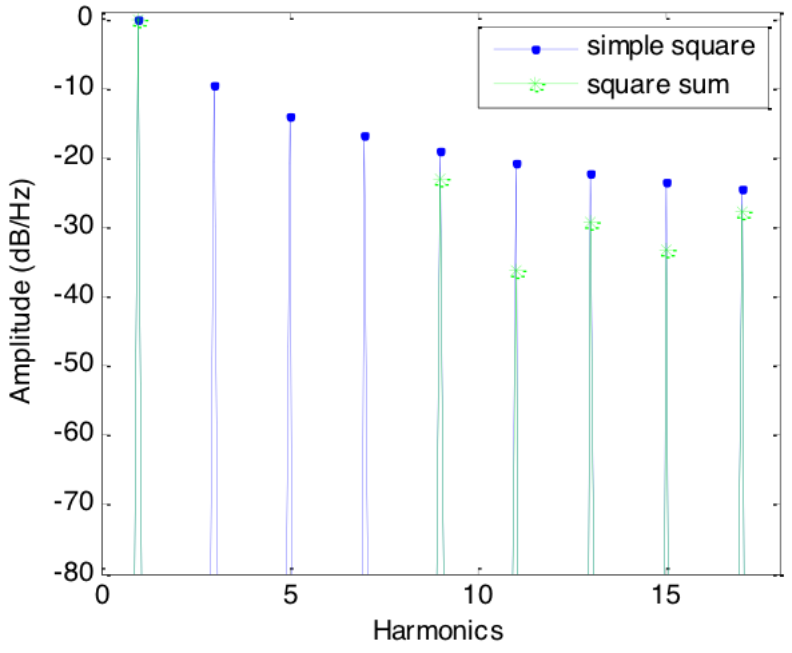

Fig. 7. Spectrum of sine-wave signal generated using square-wave signals with various phase shifts for $\mathrm{p}=2$

Regarding the two previous analytical study, we can conclude that in terms of linearity, the first approach is more 
efficient because it cancels more harmonics.

In addition, it seems that the requested accuracy of the phaseshift is very constraining. Let's consider the equation8 that provides the Fourier series coefficient affected by a phase-shift error.

$$
C_{k}(y)=\sum_{m=-p}^{p} \frac{-i}{k \pi} e^{-i k\left(\varphi_{m}+2 \pi d e v i a t i o n\right)}, \text { for k odd }
$$

Figure 8 presents the harmonic distortion ratio variation for a phase-shift deviation from $10^{-4}$ to $10^{-2}$ of a period.

As presented by Figure 8 , we observe by simulation that harmonic distortions affected by a phase-shift error of $10^{-3}$ of a period, have an amplitude of $-50 d B$.

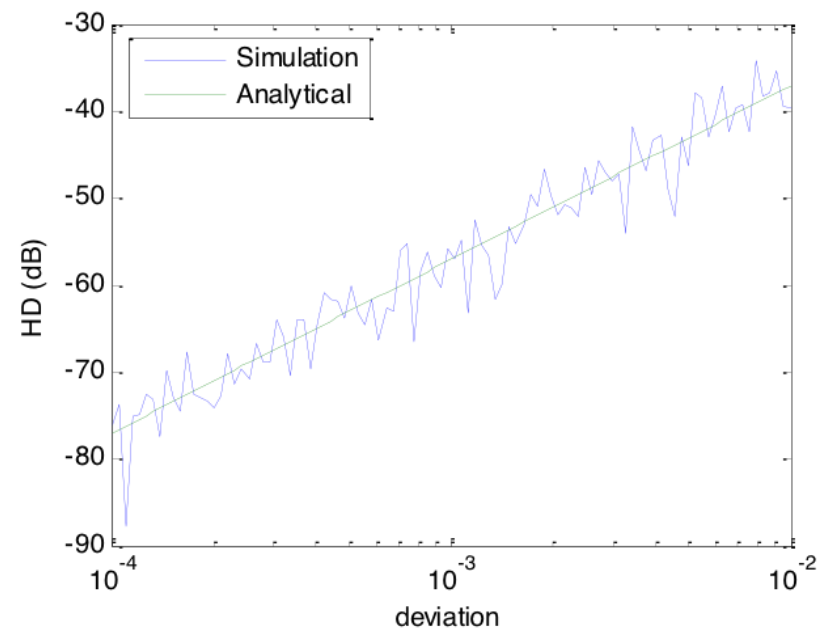

Fig. 8. Harmonic distortion ratio variations due to varying phase-shift error for a sine-wave signal generated using square-wave signals with various phase shifts for $\mathrm{p}=2$

to give an example, as illustrated by figure 8 , in order to reach a harmonic cancellation of the order of $-50 \mathrm{~dB}$ when generating a sine-wave of frequency $10 \mathrm{MHz}$, a digital frequency higher than $10 \mathrm{GHz}$ is necessary.

As mentioned in section II-A one of the parameters to vary is the number of square-wave signals to generate. The optimized number of these signals is included into the parameter $M$ of the previous analysis. $M$ is linked to the $p$ parameter. As the definition of the $p$ value is linked to the number of harmonics to cancel, the number of square-wave generators to design will be linked to the specification of the targeted application.

\section{ROBUSTNESS STUDY}

In this section, we study the impact of errors on the linearity for the generation using adjustable amplitudes of square-wave signals. The main errors are phase-shift error, jitter and gain error on amplitude.
Figure 9 presents the spectrum of the sine-wave generated with $\mathrm{p}=2$. This sine-wave signal is affected by jitter and/or gain error of the amplitude of the square-wave signals. We observe that the error on the amplitude of the square-wave induces an amplitude increase of the harmonics initially cancelled. The jitter induces an increase of the noise level.

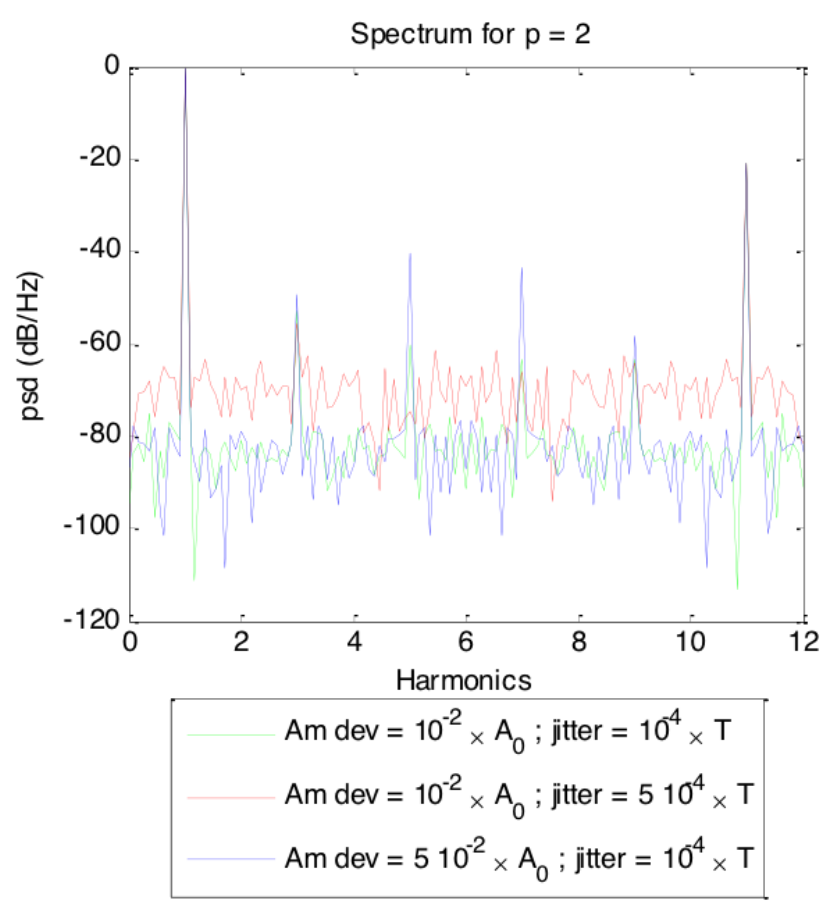

Fig. 9. Spectrum of a sine-wave signal affected by amplitude error and/or jitter on the square-wave signals for sine-wave signal generated using squarewave signals with various amplitudes for $\mathrm{p}=2$

Figure 10 presents the computation of the harmonic distortion rate for different values of jitter. The jitter has also an impact that is significant on the harmonic distortion rate. When the jitter is large, the noise floor it creates dominates the distortions due the amplitude deviation.

According to the analytical study and simulation results we propose some features for an efficient implementation. Using five sine-wave signals ( $p=2$ ), we can cancel harmonics below the eleven order. To generate $10 \mathrm{MHz}$ sine-wave signal, a $120 \mathrm{MHz}$ clock frequency is needed. The architecture with fixed phase shift and variable amplitudes is optimally demanding for its digital clock as it is a multiple of the generated frequency. In order to have harmonic distortions at a maximum of $-50 \mathrm{~dB}$, jitter should be below $0.1 \mathrm{~ns}$ and the amplitude error should be limited to $1 \%$.

\section{CONCLUSION}

This work is in the field of generating sine-wave signals by summing square-wave signals in order to cancel low-order harmonics. The effectiveness of the harmonic cancellation is related to the characteristics of the square-wave signals. Two approaches are generally implemented. The first approach consists in setting the phase shifts according to the implementation 


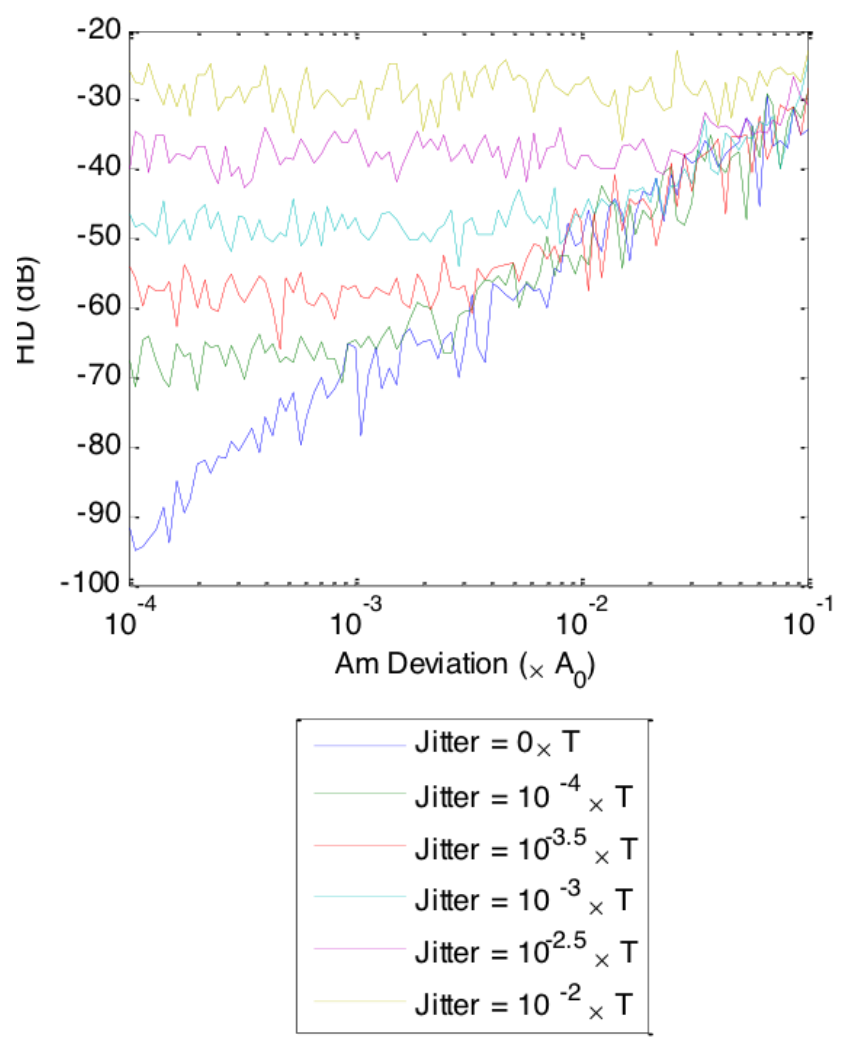

Fig. 10. Harmonic distortion ratio of a sine-wave generated using squarewave signals with various amplitudes affected by different amount of jitter

technique and setting various amplitude values of the squarewave signals. The second approach is the inverse. It consists in setting the different amplitudes at the same value and setting various phase shifts of the square-wave signals. We have analytically proven that for a given number of square-wave generators, the first approach allows to cancel a higher number of harmonics. In addition, considering targeted specifications, we have proven by simulation that the first approach is less constraining in terms of clock generation.

\section{ACKNOWLEDGMENT}

This work is part of the POPSTAR project funded by IFREMER

\section{REFERENCES}

[1] M. J. Barragan, G. Leger, D. Vazquez, and A. Rueda, "On-chip sinusoidal signal generation with harmonic cancelation for analog and mixed-signal bist applications," Analog Integrated Circuits and Signal Processing, vol. 82, no. 1, pp. 67-79, 2015. [Online]. Available: http://dx.doi.org/10.1007/s10470-014-0456-0

[2] D. Rairigh, X. Liu, C. Yang, and A. J. Mason, "Sinusoid signal generator for on-chip impedance spectroscopy," in 2009 IEEE International Symposium on Circuits and Systems, May 2009, pp. 1961-1964.

[3] M. M. Elsayed and E. Sanchez-Sinencio, "A low thd, low power, high output-swing time-mode-based tunable oscillator via digital harmoniccancellation technique," IEEE Journal of Solid-State Circuits, vol. 45, no. 5, pp. 1061-1071, May 2010.
[4] B. K. Vasan, S. K. Sudani, D. J. Chen, and R. L. Geiger, "Low-distortion sine wave generation using a novel harmonic cancellation technique," IEEE Transactions on Circuits and Systems I: Regular Papers, vol. 60, no. 5, pp. 1122-1134, May 2013.

[5] P. Aluthwala, N. Weste, A. Adams, T. Lehmann, and S. Parameswaran, "A simple digital architecture for a harmonic-cancelling sine-wave synthesizer," in 2014 IEEE International Symposium on Circuits and Systems (ISCAS), June 2014, pp. 2113-2116.

[6] C. Shi and E. Snchez-Sinencio, " $150-850 \mathrm{mhz}$ high-linearity sine-wave synthesizer architecture based on fir filter approach and sfdr optimization," IEEE Transactions on Circuits and Systems I: Regular Papers, vol. 62, no. 9, pp. 2227-2237, Sept 2015.

[7] H. Malloug, M. J. Barragan, S. Mir, E. Simeu, and H. Le-Gall, "Mostlydigital design of sinusoidal signal generators for mixed-signal bist applications using harmonic cancellation," in 2016 IEEE 21st International Mixed-Signal Testing Workshop (IMSTW), July 2016, pp. 1-6. 